PROCEEDINGS OF THE

AMERICAN MATHEMATICAL SOCIETY

Volume 133, Number 10, Pages 2865-2871

S 0002-9939(05)07845-7

Article electronically published on March 31, 2005

\title{
A VARIANT OF THE REYNOLDS OPERATOR
}

\author{
HUAH CHU, SHOU-JEN HU, AND MING-CHANG KANG
}

(Communicated by Martin Lorenz)

\begin{abstract}
Let $G$ be a linearly reductive group over a field $k$, and let $R$ be a $k$ algebra with a rational action of $G$. Given rational $R$ - $G$-modules $M$ and $N$, we define for the induced $G$-action on $\operatorname{Hom}_{R}(M, N)$ a generalized Reynolds operator, which exists even if the action on $\operatorname{Hom}_{R}(M, N)$ is not rational. Given an $R$-module homomorphism $M \rightarrow N$, it produces, in a natural way, an $R$-module homomorphism which is $G$-equivariant. We use this generalized Reynolds operator to study properties of rational $R-G$ modules. In particular, we prove that if $M$ is invariantly generated (i.e. $M=R \cdot M^{G}$ ), then $M^{G}$ is a projective (resp. flat) $R^{G}$-module provided that $M$ is a projective (resp. flat) $R$-module. We also give a criterion whether an $R$-projective (or $R$-flat) rational $R$ - $G$-module is extended from an $R^{G}$-module.
\end{abstract}

\section{$\S 1$. INTRODUCTION}

Let $G$ be a linearly reductive group over a field $k$, and let $R$ be a $k$-algebra with a rational $G$-action. Let $M$ be a rational $R$ - $G$-module. The Reynolds operator on $M$ is the canonical projection from $M$ onto the the subspace of fixed elements $M^{G}$, viewed as a linear map $M \longrightarrow M$; it is a very useful tool for studying $R$ - $G$-modules [Fo, [Ho. Given rational $R$ - $G$-modules $M$ and $N$, there is a natural $G$-action on the module $\operatorname{Hom}_{R}(M, N)$ given by $\sigma(f)(u)=\sigma\left(f\left(\sigma^{-1}(u)\right)\right)$. The fixed points for this action are precisely the $G$-equivariant $R$-module homomorphisms from $M$ to $N$. One would like to use the Reynolds operator on $\operatorname{Hom}_{R}(M, N)$ to produce from a given $R$-module homomorphism $f: M \longrightarrow N$ a $G$-equivariant $R$-module homomorphism $\pi(f): M \longrightarrow N$. This strategy was successfully used by Magid $\underline{\mathrm{Ma}}$ in case $M$ and $N$ are finitely generated projective $R$-modules, since in this case $\operatorname{Hom}_{R}(M, N)$ is a rational $G$-module; see [Ma Lemma 1]. In general, however, $\operatorname{Hom}_{R}(M, N)$ need not be a rational $G$-module (see Example 2.5 below), so the classical Reynolds operator is no longer available.

In its stead we introduce, in Section 2, a map $\pi_{M, N}: \operatorname{Hom}_{R}(M, N) \longrightarrow$ $\operatorname{Hom}_{R}(M, N)$ which turns out to be very similar to the Reynolds operator. Its main properties can be summarized as follows.

Proposition 1.1. Let $G$ be a linearly reductive group defined over a field $k$, and $R$ a k-algebra with a rational $G$-action. Suppose that $M$ and $N$ are rational $R$ $G$-modules and $\operatorname{Hom}_{R}(M, N)$ is the k-vector space of all $R$-linear maps from $M$

Received by the editors February 20, 2004 and, in revised form, May 30, 2004.

2000 Mathematics Subject Classification. Primary 13A50, 16D40, 16W22.

Key words and phrases. Rings of invariants, linearly reductive groups, Reynolds operator, rational $G$-spaces, projective modules, flat modules.

(C)2005 American Mathematical Society Reverts to public domain 28 years from publication 
into $N$. Then there is a natural idempotent map $\pi=\pi_{M, N}: \operatorname{Hom}_{R}(M, N) \longrightarrow$ $\operatorname{Hom}_{R}(M, N)$ such that

(1) for any $f \in \operatorname{Hom}_{R}(M, N), \pi(f)$ is a $G$-equivariant map;

(2) for any $R$ - $G$ module $M, N, L$, for any $f \in \operatorname{Hom}_{R}(M, N), g \in \operatorname{Hom}_{R}(N, L)$, if $f$ is $G$-equivariant, then $\pi(g \circ f)=\pi(g) \circ f$; if $g$ is $G$-equivariant, then $\pi(g \circ f)=g \circ \pi(f)$.

The proof of Proposition 1.1 is given in Section 2; see Proposition 2.7 (and Definition 3.2).

We now use the generalized Reynolds operator to study rational $R$ - $G$-modules. One of our main reults is the following theorem.

Theorem 1.2. Let $G$ be a linearly reductive group over $k$ and let $R$ be a $k$-algebra with a rational $G$-action. Suppose that $M$ is an $R$ - $G$-module such that

(1) $M=R \cdot M^{G}$, and

(2) $M$ is an $R$-projective (resp. R-flat) module.

Then $M^{G}$ is a projective (resp. flat) $R^{G}$-module.

Note that in the above theorem, the case when $M$ is a finitely generated $R$ projective module was proved by Magid [Ma, Corollary 4]; the case when $M$ is an $R$-flat module such that $G$ is a finite group with $1 /|G| \in R$ was proved by Naoum and Al-Aubaidy [NA, Theorem 2.2]. In fact, many results of [NA] can be derived from Proposition 1.1 in the same way.

We use Theorem 1.2 to prove a criterion whether an $R$ - $G$-module $M$ is extended, i.e., whether $M$ is isomorphic to $R \underset{R^{G}}{\otimes} N$ for some $R^{G}$-module, i.e.

Theorem 1.3. Let $M$ be an $R$-G-module, and define the $G$-equivariant $R$-morphism $\varphi: R \underset{R^{G}}{\otimes} M^{G} \longrightarrow M$ by $\varphi(r \otimes x)=r x$ for any $r \in R$, any $x \in M^{G}$.

(1) If $M$ is an R-projective module, then $\varphi$ is an isomorphism if and only if $M$ is invariantly generated.

(2) If $M$ is an R-flat module, then $\varphi$ is an isomorphism if and only if $M$ is strongly invariantly generated.

The term "strongly invariantly generated" will be defined in Definition 3.6.

We shall organize this note as follows. Basic constructions of Reynolds operators will be discussed in Section 2. The descent properties of rational $R$ - $G$-modules where $G$ is a linearly reductive group will be established in Section 3 and the proof of Theorem 1.2 and Theorem 1.3 will be given there.

Notation and Terminology. $G$ is a group acting on a $\operatorname{ring} R$. In case $G$ is finite with $1 /|G| \in R$, there are no other restrictions on $G$ and $R$. In case $G$ is a linearly reductive group defined over a field $k$, it is assumed tacitly that $G$ is a linear algebraic group defined over a field $k$, that $R$ is a $k$-algebra, and that $G$ acts on $R$ by $k$-algebra automorphisms. If $M$ is an $R$-G-module, the subset $M^{G}$ is defined as $M^{G}=\{m \in M: \sigma \cdot m=m$ for any $\sigma \in G\}$; in particular, $R^{G}$ is called the ring of invariants of $R$ under the action of $G$, and $M^{G}$ is a module over $R^{G}$. If $U$ and $V$ are vector spaces with $G$-actions, and $f: U \longrightarrow V$ is a linear map, $f$ is called a $G$-equivariant map (or $G$-map for short) provided $f(\sigma \cdot u)=\sigma \cdot f(u)$ for any $\sigma \in G$, any $u \in U$. 


\section{REYNOLDS OPERATORS AND GENERALIZATIONS}

We recall some basic notions of linear algebraic groups. More details can be found in $\overline{\mathrm{DC}}, \mathrm{Fo}, \mathrm{Kr}]$.

Let $k$ be any field, and $G$ a linear algebraic group defined over $k$. (It is unnecessary to assume that $k$ is algebraically closed or char $k=0$.)

Let $V$ be a vector space over $k$ with a $k$-linear $G$-action, which will be called a $G$-space. A $G$-subspace of $V$ is sometimes called a $G$-invariant subspace. (It is unnecessary to assume that $\operatorname{dim}_{k} V<\infty$.) $V$ is called a rational $G$-space if, for any $v \in V$, the set $\{\sigma \cdot v: \sigma \in G\}$ spans a finite-dimensional vector space $W$ of $V$ such that the induced map $G \rightarrow G L(W)$ is a morphism of algebraic groups.

A linear algebraic group $G$ over $k$ is called linearly reductive if every rational $G$ space is completely reducible, i.e., for any rational $G$-space $V$, and any $G$-subspace $W$ of $V$, there is a $G$-space $W^{\prime}$ of $V$ such that $V$ is $G$-isomorphic to $W \oplus W^{\prime}$.

In case $k$ is algebraically closed, the structure of linearly reductive groups is determined by Nagata [Na] (i) When char $k=0, G$ is linearly reductive if and only if the unipotent radical of $G$ is trivial, i.e., the radical of $G$ is a torus; (ii) When char $k=p>0, G$ is linearly reductive if and only if $G_{0}$ is a torus and $p \nmid\left[G: G_{0}\right]$ where $G_{0}$ is the connected component of $G$ containing the identity element. In particular, if $G$ is a finite subgroup of $G L_{n}(k)$, then $G$ is linearly reductive if and only if $\frac{1}{|G|} \in k$.

Definition 2.1. Let $V$ be a completely reducible $G$-space. Let $\rho: G \longrightarrow G L\left(V_{\rho}\right)$ be an irreducible representation of $G$. Define $V(\rho)$ to be the sum of $G$-subspaces $V^{\prime}$ of $V$ where $V^{\prime}$ runs over all $G$-subspaces which are $G$-isomorphic to $V_{\rho}$. It follows that $V=\bigoplus_{\rho} V(\rho)$ where $\rho$ runs over all inequivalent irreducible representations of $G$ [DC, pp. 73-74], $\mathrm{Kr}$ Kapitel II, 3.1 and 3.5]. $V(\rho)$ is called the $\rho$-isotypic component of $V$.

Definition 2.2. If $\rho: G \longrightarrow G L\left(V_{\rho}\right)$ is an irreducible representation and $V$ is a completely reducible $G$-space such that $V=\bigoplus_{\rho^{\prime}} V\left(\rho^{\prime}\right)$ is the isotypic decomposition, define the canonical idempotent map $\pi_{\rho}: V \longrightarrow V$ by $\pi_{\rho}(v)=v_{\rho}$ where $v=\sum_{\rho^{\prime}} v_{\rho^{\prime}}$ with $v_{\rho^{\prime}} \in V\left(\rho^{\prime}\right)$, and $V\left(\rho^{\prime}\right)$ is the unique $\rho^{\prime}$-isotypic component of $V$.

Note that, if $\rho=1_{G}$ is the trivial representation of $G$, then $\pi_{\rho}$ is the Reynolds operator of $G$; we will write $\pi_{\rho}=\pi_{0}$ in this case. From now on, we will consider $\pi_{0}$ only unless otherwise specified. We record the following facts whose proofs are omitted.

Lemma 2.3. Let $\rho: G \longrightarrow G L\left(V_{\rho}\right)$ be an irreducible representation and $V, W$ be completely reducible $G$-spaces. Suppose $\varphi: V \longrightarrow W$ is a $G$-equivariant linear map.

(1) If $v \in V(\rho)$, then $\varphi(v) \in W(\rho)$.

(2) $\varphi \circ \pi_{\rho}=\pi_{\rho} \circ \varphi$. In particular, if $\varphi: V \longrightarrow W$ is a $G$-equivariant surjective map, then the restriction map $\varphi: V(\rho) \longrightarrow W(\rho)$ is also surjective.

Definition 2.4. Let $U$ and $V$ be $G$-spaces, and $\operatorname{Hom}_{k}(U, V)$ the vector space of all linear maps from $U$ into $V$. We define a $G$-action on $\operatorname{Hom}_{k}(U, V)$ : for any $f \in \operatorname{Hom}_{k}(U, V)$, any $\sigma \in G,(\sigma f)(u):=\sigma\left(f\left(\sigma^{-1} u\right)\right)$ for any $u \in U . \quad f$ is $G$ equivariant if and only if $\sigma f=f$ for all $\sigma \in G$.

Example 2.5. If $U$ and $V$ are rational $G$-spaces, then it is not necessary that $\operatorname{Hom}_{k}(U, V)$ is a rational $G$-space. We will give an example which is suggested by the referee. 
Let $k$ be any field containing an element whose multiplicative order is infinite. Let $G=\mathbb{G}_{m}=\{a \in k: a \neq 0\}$ be the multiplicative group of $k$. Define $G$-spaces $U$ and $V$ as follows: $V=k$ and $G$ acts trivially on it; $U=k[x]$ is the infinitedimensional vector space with basis $1, x, x^{2}, \cdots$ and $a \cdot\left(\sum c_{j} x^{j}\right)=\sum c_{j} a^{-j} x^{j}$ for any $a \in G$. Consider $f \in \operatorname{Hom}_{k}(U, V)$ defined by $f\left(x^{j}\right)=1$ for any $j$. Note that $a f\left(\sum c_{j} x^{j}\right)=\sum c_{j} a^{j}$ for any $a \in G$.

Let $\alpha \in k$ be an element of infinite multiplicative order. It follows that $f, \alpha f$, $\alpha^{2} f, \cdots, \alpha^{m} f$ are linearly independent over $k$ because $\operatorname{det}\left(\alpha^{i} f\left(x^{j}\right)_{0 \leq i, j \leq m}\right) \neq 0$. Thus the dimension of the space spanned by $\{a f: a \in G\}$ is infinite.

Definition 2.6. Let $G$ be a linearly reductive group over a field $k$, and $U, V$ rational $G$-spaces. For every $f \in \operatorname{Hom}_{k}(U, V), f$ can be represented as $\lim f_{\alpha}$, the inverse limit of $f_{\alpha} \in \operatorname{Hom}_{k}\left(U_{\alpha}, V\right)$ where $U_{\alpha}$ runs over the finite-dimensional $G$-subspaces of $U$. We claim that $\operatorname{Hom}_{k}\left(U_{\alpha}, V\right)$ is a rational $G$-space. In fact, if $f \in \operatorname{Hom}_{k}\left(U_{\alpha}, V\right)$, find a finite-dimensional $G$-subspace $V_{\alpha}$ containing $f\left(U_{\alpha}\right)$. Then $\sigma f \in \operatorname{Hom}_{k}\left(U_{\alpha}, V_{\alpha}\right)$ for all $\sigma \in G$.

Thus we can define the canonical idempotent map $\pi_{0}: \operatorname{Hom}_{k}\left(U_{\alpha}, V\right) \longrightarrow$ $\operatorname{Hom}_{k}\left(U_{\alpha}, V\right)$ by Definition 2.2. Hence we can define $\pi_{0}(f)=f_{0}$ by $f_{0}=\lim _{\longleftarrow} \pi_{0}\left(f_{\alpha}\right)$. We note that, because of Lemma 2.3., $\pi_{0}(f)$ is well defined, i.e., independent of the choice of $f_{\alpha}$. It is easy to verify that $f_{0}$ is a $G$-equivariant map in $\operatorname{Hom}_{k}(U, V)$.

We remark that, if $G, U, V, f$ are the same as in Example 2.5, then $f_{0}$ is the map defined by $f_{0}\left(\sum c_{j} x^{j}\right)=c_{0}$. Let $g$ be the restriction of $f$ to the subspace $\sum_{0 \leq j \leq m} k \cdot x^{j}$. Then $g=\sum_{0 \leq j \leq m} g_{j}$ is an isotypic decomposition of $g$ where $g_{j}$ defined by $g_{j}\left(\sum c_{l} \cdot x^{l}\right)=c_{j}$ corresponds to the component of the linear character $\chi^{j}: G \rightarrow k \backslash\{0\}$ defined by $\chi^{j}(a)=a^{j}$ for any $a \in G$.

From now to the end of this section, we shall assume that $G$ is a linearly reductive group over a field $k$ and every $G$-space is a rational $G$-space.

Proposition 2.7. Let $U, V, W$ be rational $G$-spaces and $f \in H_{0}(U, V), g \in$ $H_{\text {om }}(V, W)$.

(1) If $f$ is G-equivariant, then $(g \circ f)_{0}=g_{0} \circ f$.

(2) If $g$ is $G$-equivariant, then $(g \circ f)_{0}=g \circ f_{0}$.

In other words, the Reynolds operator $\pi=\pi_{U, V}: \operatorname{Hom}_{k}(U, V) \longrightarrow \operatorname{Hom}_{k}(U, V)$ defined by $\pi(f)=f_{0}$ is natural in the following sense: If $g: V \longrightarrow W$ is $G$ equivariant and $\phi: \operatorname{Hom}_{k}(U, V) \longrightarrow \operatorname{Hom}_{k}(U, W)$ is defined as $\phi(f)=g \circ f$, then $\pi_{U, W} \circ \phi=\phi \circ \pi_{U, V}$. Similarly when $f: U \longrightarrow V$ is $G$-equivariant.

Proof. We shall prove Part (1) only. To check $(g \circ f)_{0}=g_{0} \circ f$, it suffices to compare the effects of $(g \circ f)_{0}$ and $g_{0} \circ f$ on any finite-dimensional $G$-subspaces $U^{\prime}$. For such a $U^{\prime}$, choose a finite-dimensional $G$-subspace $V^{\prime}$ such that $U^{\prime} \subset U, V^{\prime} \subset V$ and $f\left(U^{\prime}\right) \subset V^{\prime}$. In other words, we shall verify $(g \circ f)_{0}=g_{0} \circ f$ with the additional assumption that $\operatorname{dim}_{k} U<\infty$ and $\operatorname{dim}_{k} V<\infty$. Thus both $\operatorname{Hom}_{k}(U, W)$ and $\operatorname{Hom}_{k}(V, W)$ are rational $G$-spaces.

Write $g=\sum_{\rho} g_{\rho}$ where $g_{\rho} \in \operatorname{Hom}_{k}(V, W)(\rho)$. Define $\varphi: \operatorname{Hom}_{k}(V, W) \longrightarrow$ $\operatorname{Hom}_{k}(U, W)$ by $\varphi(h)=h \circ f$. Since $f$ is $G$-equivariant, thus so is $\varphi$, and $\varphi$ maps the $\rho$-isotypic component of $\operatorname{Hom}_{k}(V, W)$ into that of $\operatorname{Hom}_{k}(U, W)$ by Lemma 2.3. It follows $g_{\rho} \circ f \in \operatorname{Hom}_{k}(U, W)(\rho)$. From the equality $g_{\circ} f=\sum_{\rho}\left(g_{\rho} \circ f\right)$, we find $(g \circ f)_{0}=g_{0} \circ f$. 
The following lemma will be used in the proof of Theorem 1.2 when $M$ is $R$-flat.

Lemma 2.8. Let $U, V$ be rational $G$-spaces, $u \in U$ and $f \in H_{k}(U, V)$. Suppose that $f(\sigma \cdot u)=0$ for every $\sigma \in G$. Then $f_{0}(u)=0$.

Proof. Let $X$ be the finite-dimensional $G$-subspace generated by $\{\sigma \cdot u: \sigma \in G\}$. Let $\iota: X \longrightarrow U$ be the inclusion map. The $\iota$ is $G$-equivariant.

By Proposition 2.7, $(f \circ \iota)_{0}=f_{0} \circ \iota$. Since $f(X)=0$, it follows that $f \circ \iota=0$. Thus $f_{0} \circ \iota=0$. In particular, $f_{0}(u)=f_{0}(\iota(u))=f_{0} \circ \iota(u)=0$.

\section{3. $R$-G-MODULES}

Throughout this section, except in Theorems 3.4 and 3.5, we assume that $k$ is a field, $G$ is a linearly reductive group over $k$, acting on a $k$-algebra $R$ such that $R$ becomes a rational $G$-space. We emphasize that $R$ can be commutative or non-commutative.

Definition 3.1. An $R$-G-module $M$ is a left $R$-module with a $G$-action such that

(i) $M$ becomes a rational $G$-space, and

(ii) $\sigma(\alpha \cdot m)=\sigma(\alpha) \cdot \sigma(m)$ for any $\sigma \in G, \alpha \in R, m \in M$.

Definition 3.2. If $M$ and $N$ are $R$-G-modules, then $\operatorname{Hom}_{R}(M, N)$ is a vector space over $k$; it is a $G$-subspace of $\operatorname{Hom}_{k}(M, N)$. For each $f \in \operatorname{Hom}_{R}(M, N)$, we define $f_{0}$ as in Definition 2.6. Since $\operatorname{Hom}_{R}(M, N)$ is a $G$-subspace of $\operatorname{Hom}_{k}(M, N)$ and the Reynolds operator sends a $G$-subspace into itself, it follows that $f_{0}$ is an $R$-morphism. Note that $f_{0}$ is $G$-equivariant.

Lemma 3.3. Let $M$ and $N$ be $R$-G-modules, and $f: M \longrightarrow N$ a $G$-equivariant $R$-morphism. Suppose that, as an R-morphism, $f$ is a split epimorphism (resp. monomorphism ). Then there is a G-equivariant $R$-morphism $g: N \longrightarrow M$ such that $f \circ g=i d_{N}\left(\right.$ resp. $\left.g \circ f=i d_{M}\right)$.

Proof. We will prove only the case when $f$ is a split epimorphism. Find $g \in$ $\operatorname{Hom}_{R}(N, M)$ such that $f \circ g=i d_{N}$. Apply Proposition 1.1. We get $i d_{N}=\left(i d_{N}\right)_{0}=$ $(f \circ g)_{0}=f \circ g_{0}$. The above proof is adapted from that of [Ma Lemma 3]; in fact, the case when $M$ and $N$ are finitely generated $R$-projective modules is due to Magid.

Proof of Theorem 1.2 when $M$ is an $R$-projective module. Since $M=R \cdot M^{G}$, choose a free module $F=\bigoplus_{\lambda \in \Lambda} R \cdot e_{\lambda}$ where $\Lambda$ is some index set and choose an $R$-morphism $p: F \rightarrow M$ such that $p$ is surjective and $p\left(e_{\lambda}\right) \in M^{G}$ for any $\lambda \in \Lambda$. Define a $G$-action on $F$ by $\sigma\left(r \cdot e_{\lambda}\right)=\sigma(r) e_{\lambda}$ for any $\sigma \in G, r \in R, \lambda \in \Lambda$. Then $p$ is $G$-equivariant.

By Lemma 3.3 find a $G$-equivariant $R$-morphism $q: M \rightarrow F$ such that $p \circ q=$ $i d_{M}$.

Taking $G$-fixed points, we get $\tilde{q}: M^{G} \rightarrow F^{G}$ and $\tilde{p}: F^{G} \rightarrow M^{G}$ with $\tilde{p} \circ \tilde{q}=i d$, where $\tilde{q}$ and $\tilde{p}$ are the restriction maps of $q$ and $p$ to the $G$-fixed point sets. Note that $F^{G} \cong \bigoplus_{\lambda \in \Lambda} R^{G} e_{\lambda}$ is a free $R^{G}$-module. Hence $M^{G}$ is $R^{G}$ projective.

Before proving the remaining part of Theorem 1.2, we recall a flatness criterion of Chase [Ch, which was attributed to Villamayor. We recast [Ch, Proposition 2.2] as follows. 
Theorem 3.4. Let $R$ be any ring, and $M$ a left $R$-module. Then $M$ is a flat $R$ module if and only if, for any short exact sequence $0 \rightarrow N \stackrel{\varphi}{\rightarrow} F \stackrel{\pi}{\rightarrow} M \rightarrow 0$ where $F$ is a free $R$-module, and for any $u \in N$, there exists an R-endomorphism $\theta: F \rightarrow F$ such that $\pi \circ \theta=\pi$ and $\theta(\varphi(u))=0$.

Proof of Theorem 1.2 when $M$ is an $R$-flat module. We shall show that $M^{G}$ is $R^{G}$ flat by applying Theorem 3.4.

Let $0 \rightarrow N \stackrel{\varphi}{\rightarrow} F \stackrel{\pi}{\rightarrow} M^{G} \rightarrow 0$ be a resolution of $R^{G}$-modules and $u \in N$. Write $F=\bigoplus_{\lambda \in \Lambda} R^{G} \cdot e_{\lambda}$ where $\Lambda$ is some index set.

Let $\widetilde{F}=\bigoplus_{\lambda \in \Lambda} R \cdot e_{\lambda}$ be a free $R$-module and $\sigma\left(\alpha e_{\lambda}\right)=\sigma(\alpha) e_{\lambda}$ for any $\sigma \in G$. Then the $R$-morphism $\widetilde{\pi}: \widetilde{F} \longrightarrow M$ defined by $\widetilde{\pi}\left(e_{\lambda}\right)=\pi\left(e_{\lambda}\right)$ is a $G$-equivariant map. Since $M=R \cdot M^{G}, \widetilde{\pi}$ is surjective. Let $\widetilde{N}=\operatorname{ker}(\widetilde{\pi})$ and denote by $\widetilde{\varphi}$ the natural embedding of $\widetilde{N}$ into $\widetilde{F}$. We may regard $F$ and $N$ as subspaces of $\widetilde{F}$ and $\widetilde{N}$ respectively. Then $\pi$ and $\phi$ are restrictions of $\widetilde{\pi}$ and $\widetilde{\phi}$ respectively. Note that $\widetilde{F}^{G}=F$ and $\widetilde{N}^{G}=N$.

Since $M$ is $R$-flat, we can find by Theorem 3.4 , a $\widetilde{\theta}: \widetilde{F} \longrightarrow \widetilde{F}$ such that $\widetilde{\pi}=\widetilde{\pi}_{\circ} \widetilde{\theta}$ and $\widetilde{\theta}(u)=0$.

Let $\widetilde{\theta}_{0}$ be the homomorphism obtained from $\widetilde{\theta}$ as in Definition 3.2. Then $\widetilde{\pi}=$ $\widetilde{\pi}_{0}=(\widetilde{\pi} \circ \widetilde{\theta})_{0}=\tilde{\pi}_{0} \circ \widetilde{\theta}_{0}$ by Proposition 2.7, and $\widetilde{\theta}_{0}(u)=0$ by Lemma 2.8 because $u \in N=\widetilde{N}^{G}$. Since $\widetilde{\theta}_{0}$ is $G$-equivariant, it maps $F=\widetilde{F}^{G}$ into itself. Denoting by $\theta$ the restriction of $\widetilde{\theta}_{0}$ to $F$. It follows from Theorem 3.4 that $M^{G}$ is $R^{G}$-flat.

The following is an analogue of Theorem 1.2 when $G$ is a finite group acting on $R$ where it is unnecessary to assume that $R$ contains a field.

Theorem 3.5. Let $G$ be a finite group acting on a ring $R$ such that $1 /|G| \in R$. Let $M$ be an $R$-G-module, i.e., $\sigma(\alpha \cdot m)=\sigma(\alpha) \cdot \sigma(m)$ for $\sigma \in G, \alpha \in R, m \in M$. If $M=R \cdot M^{G}$ and $M$ is an R-projective (resp. R-flat) module, then $M^{G}$ is a projective (resp. flat) $R^{G}$-module.

Proof. For any $f \in \operatorname{Hom}_{R}(M, N)$, define $f_{0}(m)=1 /|G| \sum_{\sigma \in G} \sigma\left(f\left(\sigma^{-1} \cdot m\right)\right)$. Note that Proposition 2.7 and Lemma 2.8 are valid in the present situation. (See $\mathrm{Co}$. Lemma 2.2, pp. 227-228].) Hence the descent properties can be proved as in Theorem 1.2 .

As an application of the descent properties we study a criterion for an $R$ - $G$ module $M$ to be isomorphic to $R \underset{R^{G}}{\otimes} N$, i.e., when $M$ is extended from some $R^{G}$ module $N$.

Definition 3.6. Let $M$ be an $R$-G-module. $M$ is called invariantly generated if $M=R M^{G}$. A stronger notion than invariant generation is the case when the $R$ - $G$-module $M$ and its "relation module" are invariantly generated. Explicitly, an $R$-G-module $M$ is called strongly invariantly generated if there exist free $R$-modules $F_{2}=\bigoplus_{i \in I} R u_{i}$ and $F_{1}=\bigoplus_{j \in J} R v_{j}$ with bases $\left\{u_{i}: i \in I\right\}$ and $\left\{v_{j}: j \in J\right\}$ and $G$-equivariant $R$-morphisms $\varphi_{2}: F_{2} \longrightarrow F_{1}, \varphi_{1}: F_{1} \longrightarrow M$ such that $F_{2} \stackrel{\varphi_{2}}{\longrightarrow}$ $F_{1} \stackrel{\varphi_{1}}{\longrightarrow} M \longrightarrow 0$ is an exact sequence and $\sigma \cdot u_{i}=u_{i}, \sigma \cdot v_{j}=v_{j}$ for any $\sigma \in G$, any $i \in I$, any $j \in J$.

Proof of Theorem 1.3. (1) Use the proof of [Ma, Corollary 4]. Alternatively, for the " $\Leftarrow$ " direction, since $M$ is $R$-projective, Lemma 3.3 guarantees that $M$ is strongly invariantly generated. Apply the result of the following Part (2). 
(2) Consider the case when $M$ is a flat $R$-module.

If $\varphi$ is an isomorphism, then $M$ is invariantly generated. By Theorem 1.2. $M^{G}$ is a flat $R^{G}$-module. Choose a free $R^{G}$-module $P_{1}=\bigoplus_{j \in J} R^{G} v_{j}$ with basis $\left\{v_{j}: j \in J\right\}$ such that $\varphi_{1}: P_{1} \longrightarrow M^{G}$ is onto. Define $K=\operatorname{Ker}\left(\varphi_{1}\right)$. Then $0 \longrightarrow K \longrightarrow P_{1} \longrightarrow M^{G} \longrightarrow 0$ is an exact sequence of $R^{G}$-modules. Tensoring it with $R$, we get an exact sequence $0 \longrightarrow R \underset{R^{G}}{\otimes} K \longrightarrow R \underset{R^{G}}{\otimes} P_{1} \longrightarrow R \underset{R^{G}}{\otimes} M^{G} \longrightarrow 0$ because $M^{G}$ is flat. The $R-G$ module $R \underset{R^{G}}{\otimes} K$ is invariantly generated. Hence $M \simeq R \underset{R^{G}}{\otimes} M^{G}$ is strongly invariantly generated.

Conversely, assume that $M$ is strongly invariantly generated. Let $F_{2} \stackrel{\varphi_{2}}{\longrightarrow} F_{1} \stackrel{\varphi_{1}}{\longrightarrow}$ $M \longrightarrow 0$ be the exact sequence provided in Definition 3.6. Set $N=\operatorname{Ker}\left(\varphi_{1}\right)$. Then $N$ is an $R$-G-module. By Lemma 2.3 both $F_{2}^{G} \stackrel{\varphi_{2}}{\longrightarrow} N^{G} \longrightarrow 0$ and $0 \longrightarrow$ $N^{G} \longrightarrow F_{1}^{G} \stackrel{\varphi_{1}}{\longrightarrow} M^{G} \longrightarrow 0$ are exact sequences. Note that $F_{2}^{G} \simeq \bigoplus_{i \in I} R^{G} \cdot u_{i}$ and $F_{1}^{G} \simeq \bigoplus_{j \in J} R^{G} \cdot v_{j}$, because $\sigma \cdot u_{i}=u_{i}, \sigma \cdot v_{j}=v_{j}$ for any $\sigma \in G$. Tensoring these two exact sequences with $R$, we get exact sequences $R \underset{R^{G}}{\otimes} F_{2}^{G} \stackrel{\widetilde{\varphi}_{2}}{\longrightarrow} R \underset{R^{G}}{\otimes} N \longrightarrow 0$ and $0 \longrightarrow R \underset{R^{G}}{\otimes} N^{G} \longrightarrow R \underset{R^{G}}{\otimes} F_{1}^{G} \stackrel{\widetilde{\varphi}_{1}}{\longrightarrow} R \underset{R^{G}}{\otimes} M^{G} \longrightarrow 0$ because $M^{G}$ is a flat $R^{G}$-module by Theorem 1.2 again. Note that all the maps constructed above are functorial. Identify $R \underset{R^{G}}{\otimes} F_{2}^{G}$ with $F_{2}$ and $R \underset{R^{G}}{\otimes} F_{1}^{G}$ with $F_{1}$. Hence $\varphi: R \underset{R^{G}}{\otimes} M^{G} \longrightarrow M$ is an isomorphism.

\section{ACKNOWLEDGMENT}

We thank the referee for many constructive comments which greatly improved the presentation of this article.

\section{REFERENCES}

[Ch] S. Chase, Direct products of modules, Trans. Amer. Math. Soc. 97 (1960) 457-473. MR 0120260 (22:11017)

[Co] P. M. Cohn, Algebra, 2nd edition, vol. 2, John Wiley \& Sons, New York, 1989. MR 1006872 (91b:00001)

[DC] J. A. Dieudonné and J. B. Carrell, Invariant Theory: Old and New, Academic Press, New York, 1970. MR0279102 (43:4828)

[Fo] J. Fogarty, Invariant Theory, W. A. Benjamin, New York, 1969. MR0240104 (39:1458)

[Ho] M. Hochster, Invariant theory of commutative rings, Contemp. Math 43 (1985) 161-179. MR0810650 (87c:13009)

[Kr] H. Kraft, Geometrische Methoden in der Invariantentheorie, Vieweg, Braunschweig, 1985. MR.0768181 (86j:14006)

[Ma] A. R. Magid, Picard groups of rings of invariants, J. Pure Appl. Algebra 17 (1980) 305-311. MR.0579090 (81g:14004)

[Na] M. Nagata, Complete reducibility of rational representation of a matrix group, J. Math. Kyoto Univ. 1 (1961) 87-99. MR0142667|(26:236)

[NA] A. G. Naoum and W. K. Al-Aubaidy, Actions of finite groups on commutative rings, Tamkang J. Math. 34 (2003) 201-212. MR2001915 (2004e:13025)

Department of Mathematics, National Taiwan University, Taipei, Taiwan

Department of Mathematics, Tamkang University, Taipei, Taiwan

Department of Mathematics, National Taiwan University, Taipei, Taiwan

E-mail address: kang@math.ntu.edu.tw 